\title{
Spectroscopic Interpretation: The High Vibrations of $\mathrm{CDBrClF}$
}

\author{
C. Jung , C. Mejia-Monasterio ${ }^{1}$ \\ Centro de Ciencias Fisicas, UNAM \\ Av. Universidad, 62251 Cuernavaca, Mexico \\ and \\ H. S. Taylor \\ Department of Chemistry \\ University of Southern California \\ Los Angeles, California 90089
}

\begin{abstract}
We extract the dynamics implicit in an algebraic fitted model Hamiltonian for the deuterium chromophore's vibrational motion in the molecule $\mathrm{CDBrClF}$. The original model has 4 degrees of freedom, three positions and one representing interbond couplings. A conserved polyad allows in a semiclassical approach the reduction to 3 degrees of freedom. For most quantum states we can identify the underlying motion that when quantized gives the said state. Most of the classifications, identifications and assignments are done by visual inspection of the already available wave function semiclassically transformed from the number representation to a representation on the reduced dimension toroidal configuration space corresponding to the classical action and angle variables. The concentration of the wave function density to lower dimensional subsets centered on idealized simple lower dimensional organizing structures and the behavior of the phase along such organizing centers already reveals the atomic motion. Extremely little computational work is needed.
\end{abstract}

${ }^{1}$ present address: Center for Nonlinear and Complex Systems, Universita dell' Insubria, Via Vallegio 11, 22100 Como, Italy 


\section{Introduction}

In recent years we have developed methods to investigate algebraic models ( spectroscopic Hamiltonians ) for the vibrations of molecules [1, 2, 3, 4, 5]. The Hamiltonians reproduce and encode by their construction the experimental data [6, 7, 8, 9, 10, 11, 12]. So far we have treated examples where the existence of conserved quantities allowed the reduction of the system from four or three degrees of freedom to two degrees of freedom. In the present paper we treat for the first time an example, which only can be reduced to three degrees of freedom. We show to which extent our methods still work as before and how they reveal the dynamics of the deuterium in $\mathrm{CDBrClF}$ [13].

For the already known wave function ( they are needed to construct the Hamiltonian ) transformed from the number representation so as to be able to be plotted in the toroidal configuration space of action/angle variables [14] we show that we can visually sort most of the states into ladders of states with similar topology. Each ladder has a relatively simple spectrum. Complexity arises from their interleaving. We also can recognize underlying classical lower dimensional organizing structures by the fact that for these inherently complex functions the density ( magnitude squared ) is concentrated around them and the phase has simple behavior near them. The position of these structures in angle space reveals the nature of the resonance interaction causing the topology. It also allows the reconstruction, often by analytic use of the transformation back to generalized coordinates, which are near displacement coordinates, of the motion of the atoms which underlie this particular quantum state. By counting nodes in plots of the wave function density and phase advances in corresponding plots of the phase quantum excitation numbers can be obtained. In many cases, along with the polyad quantum number itself, we thereby obtain a complete set of quantum numbers for a state even though the corresponding classical motion is nonintegrable. These classification numbers can be interpreted as quasi conserved quantities for this particular state or for the ladder of states based on the same dynamic organizational element. Note there are usually several different types of organizing structures and hence ladders in the same energy region.

Why are the wave functions that are so visually complex as to be unsortable in displacement coordinate space simple enough to be sorted in our semiclassical representation ? The answer is fivefold. First, when a spectroscopic Hamiltonian is used and as energy changes frequencies can move into resonance, that is rational ratio, a constant of the motion called the polyad number comes into existence ( see section 2 ). It can be used to reduce the dimension of the configuration space when the action angle variables, which come out in taking the semiclassical limit of the spectroscopic Hamiltonian, are used. Dimension reduction is always a great simplification. Second, the resonant form of the fitted spectroscopic Hamiltonian can be justified formally by using Van Vleck or canonical perturbation theory starting with a given potential surface [13, 15, 16] in a way that takes in only the dominant dynamics. The terms that are left out for the most part are multiple small perturbations that in turn cause multiple small distortions to the wave function. This makes them harder to recognize but not fundamentally different. An excellent example of this is seen in [9], section 3 and quite 
pictorially in [17] where figures 4 and 9 show the wave functions for the same states but computed from the full and the spectroscopic Hamiltonian respectively. The simplicity of the latter is enough to illustrate our point. The third reason for the relative wave function simplicity is that as the bound wave functions lie on a toroidal configuration space their parts must interfere constructively as each angle is changed by $2 \pi$. This coherence results in simpler but not always simple wave functions. The fourth reason is in the freedom to choose a particular type of reduced dimension canonical variables. These variables are designed with guidance from the polyad and the angle dependence of the resonance to have zero velocity when the phase locking caused by the resonance sets in. The wave function in reduced configuration space will then in turn further collapse onto the environs of ( in the sense of small oscillations about ) even lower dimensional surfaces or lines. We defer explaining and exhibiting this point till section 3 which introduces needed notation. The fifth reason for simplicity is that, as alluded to above, the wave function is complex and can then be plotted separately in density and phase. Each part helps in visually sorting of wave function topologies and therefore dynamics and each yields the values of different quantum numbers.

As organizational elements we do not necessarily use actual classical periodic orbits. In many cases it is simpler to study the general ( average ) classical flow and to relate to it some idealized guiding center and to use this idealized motion as the skeletal element on which the quantum density is concentrated. We can imagine that this idealized structure contains the essence of a large number of periodic orbits performing on average a similar motion. The quantum dynamics is not able to resolve all the fine details of the classical motion, nor the infinity of long periodic orbits, nor their enormous number of bifurcations. Its limited resolution only recognizes the average of the classical dynamics over cells of the size of its resolution. Therefore we interpret the quantum state as based on the motion of the atoms which corresponds to the idealized structural element. In many cases one state of the group of similarly organized states can be interpreted as the quantum ground state on this element and the other ones are various longitudinal and transverse excitations of the basic structure, hence the ladders. In some cases only sections of a ladder are found.

Further in this paper, we see how the higher number and smaller spacing between observed states of a system with more degrees of freedom makes the whole investigation more complicated and in particular how the higher density causes more mixing of structures. This increase of structural mixing might be interpreted as one way in which effects of classical chaos enter the quantum behavior and results in a group of states being unassignable even in the semiclassical angle representation that brought simplicity in appearance to the density and phases of the assignable states.

In section 2 we discuss the spectroscopic Hamiltonian, section 3 presents the semiclassical representation of the wave functions on the toroidal configuration space and section 4 contains the dimensional reduction. Section 5 explains the basic ideas how to extract the assignment of a wave function from its plot on the reduced configuration torus. Section 6 explains how from information about the dynamics in the reduced system we go back to reconstruct information about the original system. Section 7 contains the classification of states and section 8 gives final remarks. 


\section{The model}

For the description of the chromophore dynamics of the molecule CDBrClF we use the model set up in [13]. It is based on 4 degrees of freedom as required by the nature of the observed overtone spectroscopy. They describe the three degrees of freedom of the $\mathrm{D}$ atom (index $\mathrm{s}$ for the stretch and indices $\mathrm{a}$ and $\mathrm{b}$ for two bends, where index $\mathrm{a}$ is for the bend in the C-D-F plane and index $\mathrm{b}$ for the bend out of this plane ) and the C-F stretch ( index f ). The algebraic Hamiltonian fitted to experiment and to calculations on a fitted potential surface has a natural decomposition

$$
H_{q}=H_{0}+W
$$

into a diagonal part

$$
H_{0}=\sum_{j} \omega_{j} a_{j}^{\dagger} a_{j}+\sum_{j \leq m} x_{j, m} a_{j}^{\dagger} a_{j} a_{m}^{\dagger} a_{m}
$$

(here the indices $\mathrm{j}, \mathrm{m}$ run over the 4 degrees of freedom $\mathrm{a}, \mathrm{b}, \mathrm{f}, \mathrm{s}$ ) and an interaction part

$$
\begin{gathered}
W=\sum_{j, m} k_{s, j, m}\left[a_{s} a_{j}^{\dagger} a_{m}^{\dagger}+a_{s}^{\dagger} a_{j} a_{m}\right] /(2 \sqrt{2})+ \\
\gamma\left[a_{a} a_{a} a_{b}^{\dagger} a_{b}^{\dagger}+a_{a}^{\dagger} a_{a}^{\dagger} a_{b} a_{b}\right] / 2+ \\
\delta\left[a_{a} a_{a} a_{f}^{\dagger} a_{f}^{\dagger}+a_{a}^{\dagger} a_{a}^{\dagger} a_{f} a_{f}\right] / 2+ \\
\epsilon\left[a_{b} a_{b} a_{f}^{\dagger} a_{f}^{\dagger}+a_{b}^{\dagger} a_{b}^{\dagger} a_{f} a_{f}\right] / 2
\end{gathered}
$$

where $a_{j}$ and $a_{j}^{\dagger}$ are the usual harmonic destruction and creation operators of degree of freedom j. In Eq. 3 the indices $\mathrm{j}$ and $\mathrm{m}$ run over the 3 degrees of freedom $\mathrm{a}, \mathrm{b}, \mathrm{f}$. For the coefficients we use the ones given in column 4 in table VIII in [13] where we see that roughly $\omega_{s} \approx 2 \omega_{a} \approx 2 \omega_{b} \approx 2 \omega_{f}$ giving rise to the Fermi resonances anticipated by the terms of Eq.3 and the Darling-Dennison resonances anticipated by the terms of Eqs.4-6. Eq.2 has first an "oscillator" term and a second "anharmonic" Dunham term.

The implied unperturbed basis of the space upon which $H_{q}$ operates is the number basis

$$
|\vec{n}>=| n_{s}, n_{f}, n_{a}, n_{b}>=\left|n_{s}>\right| n_{f}>\left|n_{a}>\right| n_{b}>
$$

$n_{j}$ are the quantum numbers or degree of excitation of the harmonic mode $j$ and are eigenvalues of the number operator $\hat{n}_{j}=a_{j}^{\dagger} a_{j} . \mid n_{j}>$ is its pure oscillator mode eigenfunction. $H_{0}$ is diagonal on this basis. The unperturbed ( first term of $H_{0}$ ) energy of a basis function $|\vec{n}\rangle$ is $E_{\vec{n}}^{0}=\sum_{j} n_{j} \omega_{j}$. When frequencies are near resonant as $\omega_{s} \approx 2 \omega_{a} \approx 2 \omega_{b} \approx 2 \omega_{f}$ a polyad naturally arises with many consequences. First we can write $E_{\vec{n}}^{0}=2 P \omega_{b}$ with the polyad $\mathrm{P}$ defined as

$$
P=n_{s}+\left(n_{f}+n_{a}+n_{b}\right) / 2
$$

with the corresponding operator given as $\hat{P}$ by replacing $n_{j} \rightarrow \hat{n}_{j}$ in Eq.8. Clearly all basis functions with $n_{j}$ giving the same value of $\mathrm{P}$ are degenerate. If basis functions 
are sorted by polyad, the Hamiltonian matrix which can be evaluated algebraically, is expected by perturbation theory ( degenerate basis functions interact most strongly ) to be nearly block diagonal suggesting that $P$ is a near constant of motion. In fact, it is totally block diagonal as $P$ is here an exact constant of the motion since $\hat{P}$ commutes with $H_{q}$ by construction [13, albeit not with the true Hamiltonian to which $H_{q}$ is, as said, to a high order of perturbation an approximation. When each block is diagonalized, each eigenfunction

$$
\left|\Psi_{j}^{P}>=\sum_{\vec{n} \in P} c_{\vec{n}}^{j}\right| \vec{n}>
$$

in block $\mathrm{P}$ has a good conserved quantum number $\mathrm{P}$ which roughly measures via the equation for $E_{\vec{n}}^{0}$ the degree of excitation of the state in units of the lowest frequency. One can now study each polyad separately ( second consequence of $P$ ). Here we will concentrate on the one 161 state polyad $P=5$ which is the most complex one in the range of measured energy.

\section{Semiclassical representation}

Having the $\left\{c_{\vec{n}}^{j}\right\}$ now allows us to immediately obtain, without any extra work, semiclassical eigenfunctions in action/angle variables ( good to the lowest two orders in $\hbar$ ). We do this because as we will see such eigenfunctions can be viewed due to the existence of the polyad as effectively having one less dimension. Hence the problem must now be transformed from the number representation to a semiclassical action/angle representation. The drop in dimension is characteristic of classical mechanics where a constant of the motion, here the classical analogue of $P$, can by canonical transformation allow one to reduce the dimension of the phase space. Here the reduction is from a four degree of freedom system with action/angle coordinates $\vec{I}, \vec{\phi}$ to a reduced system with specific value of $P$ and three degrees of freedom with action/angle coordinates $\vec{J}$, $\vec{\psi}$. This of course will accomplish the first promised simplification, that is dimension reduction.

To make a transition to the corresponding classical Hamiltonian we bring the operators in $H_{q}$ first into symmetrical order, and then apply the semiclassical substitution rules

$$
a_{j} \rightarrow I_{j}^{1 / 2} \exp \left(-i \phi_{j}\right), a_{j}^{\dagger} \rightarrow I_{j}^{1 / 2} \exp \left(i \phi_{j}\right)
$$

where $I_{j}$ and $\phi_{j}$ are the classical action and angle variables. The resulting classical Hamiltonian is using Eqs.2-6

$$
\begin{gathered}
H_{0}=\sum_{j}\left(I_{j}-1 / 2\right) \omega_{j}+\sum_{j \leq m} x_{j, m}\left(I_{j}-1 / 2\right)\left(I_{m}-1 / 2\right) \\
W=\sum_{j, m} k_{s, j, m} I_{s}^{1 / 2} I_{j}^{1 / 2} I_{m}^{1 / 2} \cos \left(\phi_{s}-\phi_{j}-\phi_{m}\right) / \sqrt{2}+ \\
\gamma I_{a} I_{b} \cos \left(2 \phi_{a}-2 \phi_{b}\right)+
\end{gathered}
$$




$$
\begin{gathered}
\delta I_{a} I_{f} \cos \left(2 \phi_{a}-2 \phi_{f}\right)+ \\
\epsilon I_{b} I_{f} \cos \left(2 \phi_{b}-2 \phi_{f}\right)
\end{gathered}
$$

It has the conserved quantity

$$
K=I_{s}+\left[I_{a}+I_{b}+I_{f}\right] / 2
$$

Because of the symmetric ordering required for the transition to classical dynamics the zero point between the eigenvalues of $P$ and the corresponding value of $K$ is shifted by $5 / 4$.

The reader, as an aside, might wish to contemplate how easily this transition was taken. For the more familiar $H=T+V$ Hamiltonian form one must solve the HamiltonJacobi equation to get such variables; indeed a difficult process even if the system is completely integrable. The simplicity is due to the fact that, as said, this should be rigorously done starting with a potential surface and using perturbation theory. A price is paid for circumventing perturbation theory and using a fitted spectroscopic Hamiltonian. This price is that the relation of the $a_{j}^{\dagger}$ and $a_{j}$ to the generalized momentum and position variables $p_{j}$ and $q_{j}$ is rigorously unknown. We will return to this issue later when going back from reduced space to displacement coordinates; our so called lift process.

If one now quantizes any single harmonic oscillator term in Eqs.11-15 using Schroedinger correspondence $I_{j} \rightarrow-i \partial / \partial \phi_{j}, \phi_{j} \rightarrow \phi_{j}$ it is clear that, since the angles define a now toroidal configuration space,

$$
\left|n_{j}\right\rangle \rightarrow \exp \left(i n_{j} \phi_{j}\right),|\vec{n}\rangle \rightarrow \exp (i \vec{n} \cdot \vec{\phi})
$$

with $n_{j}$ corresponding to $I_{j}-1 / 2$. From Eq.9 we get

$$
\Psi_{j}^{P} \rightarrow \Psi_{j}^{P}(\vec{\phi})=\sum_{\vec{n} \in P} c_{\vec{n}}^{j} \exp (i \vec{n} \cdot \vec{\phi})
$$

That is, the semiclassical wave functions are already known and given by Eq.18.

\section{Dimension reduction}

Dimension reduction can now be carried out by noting that here, using the polyad of Eq.8 to eliminate the fast degree of freedom $\mathrm{s}$, that the $\mathrm{j}$-th eigenstate can be written

$$
\begin{gathered}
\Psi_{j}^{P(\vec{\phi})=} \exp \left(i P \phi_{s}\right) \sum_{n_{a}, n_{b}, n_{f}} c_{P, n_{a}, n_{b}, n_{f}}^{j} \prod_{k=a, b, f} \exp \left(i n_{k}\left(\phi_{k}-\phi_{s} / 2\right)\right) \\
=\exp \left(i P \phi_{s}\right) \sum_{n_{a}, n_{b}, n_{f}} c_{P, n_{a}, n_{b}, n_{f}}^{j} \prod_{k=a, b, f} \exp \left(i n_{k} \psi_{k}\right) \\
=\exp \left(i P \phi_{s}\right) \chi_{j}^{P}\left(\psi_{a}, \psi_{b}, \psi_{f}\right)
\end{gathered}
$$


This shows that the eigenfunctions in a given polyad $P$ have a common phase factor dependence on $\phi_{s}$ and really only depend on $\chi_{j}^{P}$ which is a function of the three angles

$$
\psi_{j}=\phi_{j}-\phi_{s} / 2
$$

for $j=a, b, f$. These three angle make up a three dimensional toroidal configuration space $T^{3}$ upon which $\chi_{k}^{P}$ is situated. In fact $\chi_{k}^{P}$ is just the Fourier decomposition of the reduced dimension wave function on the configuration torus. More rigorously the wave function $\chi_{j}^{P}$ defined by Eqs.19- 21 actually is the semiclassical wave function after the classical constant of motion $K$ is used to reduce dimension by a canonical transformation. Using the angle transformation of Eq.22 suggested above by the existence of $P$ plus the trivial transformation

$$
\theta=\phi_{s}
$$

the canonical transformation permits that the new actions are $K$, given by Eq.16 and

$$
J_{j}=I_{j}
$$

again for $j=a, b, f$. $\theta$ is the cyclic angle. Staring with equations 11 to 15 and using equations $16,11-24$ a new Hamiltonian in the six canonical variables $\vec{J}, \vec{\psi}$ albeit parametric in $P$ or $K$ can now be written so directly that to save space we omit it. The torus $T^{3}$ is the configuration space. Using the Schroedinger quantization for the new variables is only semiclassical, i.e. is correct in the two lowest orders in $\hbar$. Its use gives the semiclassical expression

$$
\chi_{j}^{P}\left(\psi_{a}, \psi_{b}, \psi_{f}\right)=\sum_{n_{a}, n_{b}, n_{f}} c_{P, n_{f}, n_{a}, n_{b}}^{j} \exp \left(i\left(n_{a} \psi_{a}+n_{b} \psi_{b}+n_{f} \psi_{f}\right)\right)
$$

for the eigenfunctions on the reduced configuration space.

In the new coordinates the basis functions with polyad number $P$ and three indices $n_{f}, n_{a}, n_{b}$ corresponds to the product of three plane waves or rotors as

$$
\prod_{k=a, b, f} \exp \left(i n_{k} \psi_{k}\right)
$$

Each single plane wave factor loops the $T^{3}$ in the positive direction. The rotor is that into which the single mode harmonic oscillator has been transformed. Since Eq. 25 is a manifestly complex function it can be written as

$$
\Psi_{j}^{P}(\vec{\psi})=\left|\Psi_{j}^{P}(\vec{\psi})\right| e^{i \Phi_{j}^{P}(\vec{\psi})}
$$

where the phase function $\Phi_{j}^{P}(\vec{\psi})$ is given as

$$
\Phi_{j}^{P}=\arctan \left(\operatorname{Im} \Psi_{j}^{P} / \operatorname{Re} \Psi_{j}^{P}\right)
$$

For the basis function of Eq.26 it is well to note for future reference that the density is constant and the phase is

$$
\Phi_{\vec{n}}=n_{f} \psi_{f}+n_{a} \psi_{a}+n_{b} \psi_{b}
$$


Symmetry properties of the system in the toroidal configuration space will be used in the following. The Hamiltonian is invariant under a simultaneous shift of all angles $\psi_{j}$ by $\pi$. Therefore the reduced configuration torus $T^{3}$ covers the original configuration space twice and all structures show up in double, even though they really exist only once. In addition the system is invariant under a shift of any angle $\psi_{j}$ by $2 \pi$ and it is invariant under a reflection where all angles go over into their negatives.

\section{Methods to evaluate wave function plots}

It is seen that the simple basis functions have constant density and any eigenstate dominated by a single basis function has a density without sharp localization. Resonances as for example $2 \omega_{a} \approx \omega_{s}$ will be seen to cause localization about a line $\psi_{a}=$ constant. This follows as $\psi_{a}=\phi_{a}-\psi_{s} / 2=$ constant when differentiated with respect to time gives the frequency relation. Hence it is seen that resonances are associated with localization and the fact that $d \psi_{j} / d t=0$. It can be claimed that by using angle coordinates that slow to zero velocity at resonance we here assure that the wave function will "collapse" onto and about a lower dimensional subspace called the organization center. This in reverse gives a way to recognize the influence of resonances, namely localization of the wave function on the configuration torus. Each linearly independent locking of angles therefore reduces the dimension of the subset of configuration space in which the wave function is concentrated by 1 dimension.

The fact that the wave function is on $T^{3}$, a three dimensional torus, presents graphical and visual challenges when attempting to sort wave functions by inspection. To meet these challenges we employ the fact that $T^{3}$ is 1:1 with a cube with sides of angle range $2 \pi$ and identified opposite boundary points. That is a point on one surface of the cube is the same as a point on the direct opposite side of the cube. The density and phase are plotted in such cubes. The density plots now over the interior of the cube are organized over the whole cube or about interior planes or lines or points if zero, one, two or three independent resonant couplings respectively are active. Over (or "along" in the case of a line) the organizing structure a smooth density with no sharp localization should appear.

Nodal structures will be visible and countable in directions perpendicular to the organization element and clearly will be associated with a localized direction. The count of such transversal nodes supplies for each direction of localization a transverse quantum number $t$, that replaces an original mode quantum number $n$ which has been destroyed by the resonant interaction. The wave function in all the localized directions can be considered as qualitatively similar ( a continuous deformation ) of an oscillator state of the corresponding dimension. By the superposition of the running wave basis functions with appropriate weights and signs locally around the organization center the nodal pattern of an oscillator is reproduced. The transverse quantum numbers $t_{k}$ are the corresponding oscillator excitation numbers.

The phase functions $\Phi$ according to Eq.28 are smooth and close to a plane wave in the neighborhood of the organization center. They tend to have jumps by $\pi$ and 
singularities away from the center. Therefore phase advances along fundamental cycles ( loops on the torus or lines that connect opposite boundary points on the cube ) of the organization center are well defined and are necessarily integer multiples of $2 \pi$. They provide longitudinal quantum numbers $l_{k}$ which also replace the interacting mode quantum numbers.

Some states present what at first look appears to be a familiar but unexpected phase pattern in that the phase picture is too simple. Cases will appear when the density is localized but the phase picture has the globally striped flag appearance of the nearly noninteracting mode case. The strangeness disappears if ideas from nonlinear dynamics are employed. Nonlinear dynamics tells us that at low $P$ most of the motion takes place on what is called the primary zone of phase space. And the now interacting states are quantization of tori in this region. This zone has no central organizing structure other than the whole configuration space itself. It does contain quasiperiodic orbits corresponding to the uncoupled modes.

As $P$ changes coupling of a subset or even all modes can occur by at least two mechanisms. First is one where the existing mode periodic orbits bifurcate and create new secondary zones based with newly created organizing structures ( periodic orbits, 2 tori, 3 tori e.t.c. ). Also new secondary zones may be created seemingly spontaneously due to what are called saddle-center bifurcations. In these secondary cases the motions will have changed and states quantized based on tori or near tori ( think about the resolution of $\hbar)$ will have density and phase diagrams as described above with $\left(P, t_{k}, l_{k}\right)$ quantum numbers.

The second mechanism of coupling which will manifest itself in density localization is by continuous deformation; a process visualized by mentally drawing the primary configuration on rubber sheets and stretching and pulling the sheets in various directions till the localized form is achieved. Under such continuous deformation the phase function $\Phi$ of the continuous, single-valued complex wave function $\Psi(\vec{\psi})$ always must change by an integer multiple of $2 \pi$ as its argument $\vec{\psi}$ goes around any closed loop $\gamma$ in configuration space. In our case prior to deformation $\Phi=\vec{n} \cdot \vec{\psi}$ and that integer is $n_{j}$ if the loop is in $\psi_{j}$ ( this loop will be called $\gamma_{j}$ ) i.e. as $\psi_{j} \rightarrow \psi_{j}+2 \pi, \Phi \rightarrow \Phi+n_{j} 2 \pi$. More generally for any $\Phi$ this can be stated as the loop integral relation

$$
m_{j}(\lambda)=\frac{1}{2 \pi} \int_{\gamma_{j}} d \Phi(\vec{\psi} ; \lambda)
$$

where $m_{j}$ is an integer and $\lambda$ is the deformation parameter. Now under a continuous deformation it is clear that $m_{j}$ or in our case $n_{j}$ must remain constant as a continuous integer valued function. In our case this $n_{j}$ can be read off from the phase diagram as equal to the number of revolutions of the phase along the loop $\gamma_{j}$ achieved as $\psi_{j} \rightarrow$ $\psi_{j}+2 \pi$. For the noninteracting modes the afore mentioned quantum number $l$ is just the $n$.

From the point of view of basis state mixing even though the phase is behaving as if a single basis function is totally dominant, significant state mixing can occur. This can and will occur in proportion to the amount of localization of the density. Weak 
localization tends to occur when one basis function is dominant, the one with the same $\vec{n}$.

The $\vec{n}$ assignment holds till a small change in $\lambda$ causes an abrupt change in $\Phi$, that is when singularities occur. At this point continuous deformation will stop. Now since the wave function must be continuous, singularities in phase can only occur where the density and hence the wave function is zero. For us this occurs at the nodes and perhaps the outer edges of the localized oscillator. In short when nodes occur one can no longer use this simple full set of $\vec{n}$ quantum numbers. One can still count phase revolutions along loops inside an organized region ( parallel to the organizing center ) to get the $l$ quantum numbers. Great care should be exercised in deciding if for a localized density the phase is a continuation of a plane wave or not. To do this $\Phi(\vec{\psi})$ must be a smooth function in the neighborhood of the loop along which one is counting the phase revolutions.

\section{$6 \quad$ Ladders and motions in displacement coordinates}

Once the wave functions in their semiclassical reduced dimension representation are diagrammatically presented and quantum numbers assigned as described in the previous section, as will be seen in the next section, most often states can be visually sorted and ordered into ladders of states with a given topology. Each ladder tends to lie in the Husimi sense in one of the phase space resonance zones discussed previously. The simplicity of sorting is due to the fact that as said an idealized classical organizing element underlies each ladder. This structure can easily be written as a configuration space relation between the $\vec{\psi}$ variables. The structure will allow us to immediately determine what type of resonance interaction is creating this structure. An approximate procedure that transforms the variables $\vec{\psi}$ back to displacement space reveals the atomic motion ( modes of motion ) whose quantization yields the ladders of states. This process we call "lifting" and refer to it as the "lift". We now describe the lift in a way that starts with the least approximate and most difficult to implement algorithm and proceeds to simpler to implement ones that still retain the qualitative spirit of the motions.

To determine the key interactions we only illustrate the procedure with a simple example. Assume that the organization structure is a line (or fiber) along $\psi_{a}=0$. Since $\psi_{a}=\phi_{a}-\phi_{s} / 2$ then $\dot{\psi}_{a}-\dot{\phi}_{s} / 2 \approx \omega_{a}-\omega_{s} / 2 \approx 0$ and the $2 \omega_{a}=\omega_{s}$ phase locking relation speaks to us of a 2:1 Fermi resonance between the bending mode a and the stretch mode s. Other cases are similarly treated.

Now for interpretation of the motion a trajectory in the $(\vec{J}, \vec{\psi})$ phase space need be found which when projected into configuration space should represent the organizing element. In reverse the knowledge of the organizing element is helpful in finding an appropriate trajectory. To start this search estimates of the actions will be useful. For uncoupled modes the assigned $n_{j}$ quantum numbers using $I_{j}=n_{j}+1 / 2$ suffices. To obtain estimates of the initial actions the quantum mechanical average of the $J_{j}$ can 
be used and trivially computed from the known wave functions as

$$
<J_{j}>=<\Psi\left|-i \frac{\partial}{\partial \psi_{j}}\right| \Psi>=\sum\left|c_{\vec{n}}\right|^{2} n_{j}
$$

The ladder state that is most localized should be used for this and to check an estimate the variance can be used. With this we now have initial conditions $\left(<J_{j}\right\rangle$ and a value of $\psi_{j}$ on the organizing structure) with which to uses Hamilton's equations to get $\psi_{j}(t), J_{j}(t)$. Then first we reconstruct the cyclic angle $\theta$ by integrating

$$
\theta(t)=\theta(0)+\int_{0}^{t} d s \frac{\partial H}{\partial K}\left(\psi_{j}(s), J_{j}(s)\right)
$$

Next we undo the canonical transformation to get the corresponding $\phi_{j}(t)$ and $I_{j}(t)$. Then we assume some idealized harmonic model for the elementary degrees of freedom and form the displacement $q_{j}(t)$ and its conjugate momentum $p_{j}(t)$ as

$$
q_{j}(t)=\sqrt{2 I_{j}(t)} \cos \left(\phi_{j}(t)\right), p_{j}(t)=\sqrt{2 I_{j}(t)} \sin \left(\phi_{j}(t)\right)
$$

The functions $q_{j}(t)$ will be interpreted as representatives of the atomic motion.

Diagrams in which the $q_{j}$ are taken as coordinates with $\mathrm{t}$ as parameter give the more conventional picture of the atomic motions. In the simplified methods the average values of $I_{j}$ which giving the scale of the $q_{j}$ motion will replace $I(t)$ in Eq.33. These methods are simple because again Eq.33 is used but the time dependence and therefore the computation is suppressed and the relations gotten between the $q_{j}$ from the organizing structure equation that relates $\phi_{j}$ along with the use of Eqs.23, 24.

In preparation for section 7 let us discuss a few very simple cases. First there is the possibility that the density is distributed rather homogeneously over the whole $T^{3}$ and the phase function comes close to a global plane wave with 3 component wave vector $\left(n_{a}, n_{b}, n_{f}\right)$. This means no locking at all, all modes run freely with their individual frequency and the excitation numbers of the various modes are $n_{j}$ read off from the wave vector for $j=a, b, f$ (i.e. count the revolutions as one traverses the phase diagram in the $\mathrm{j}$ 'th direction) and $n_{s}=P-\left[n_{a}+n_{b}+n_{f}\right] / 2$. If the density is concentrated in a plane, e.g. the plane $\psi_{a}=$ constant then the angle $\psi_{a}$ does not move in the long run. It only may oscillate around the value of the constant. ¿From the canonical transformation of Eq.22 we see that this means $\phi_{a}-\phi_{s} / 2=$ constant for the angles of the original degrees of freedom. Therefore we have frequency and phase locking between the modes a and $s$. The other modes $b$ and $f$ run freely and their excitation is according to the wave vector of the phase function in the plane of high density. Analogous considerations hold for other planes of concentration of the density. If the density is concentrated along a line, e.g. the line $\psi_{a}=$ constant and $\psi_{b}=$ constant ( this is a fiber running in $\mathrm{f}$ direction ) then we see from Eq.22 that $\phi_{a}-\phi_{s} / 2$ and $\phi_{b}-\phi_{s} / 2$ are kept close to constant values and this means locking between a and $\mathrm{s}$ and simultaneously locking between $\mathrm{b}$ and s. Of course, then also a and b are locked relatively to each other. The mode $f$ runs freely in this case and its excitation number follows from the wave number of the phase function along the fiber of high density. Analogous considerations will be applied for a concentration of the density along other 1 dimensional lines in reduced configuration space. 


\section{The motion behind individual quantum states}

For polyad 5 with 161 states, the given transformations and equations lead to a three dimensional toroidal configuration space which here is a cube with coordinates $\psi_{a}, \psi_{b}$ and $\psi_{f}$. Three dimensional plots of the semiclassical state functions look too often like large globes of ink and were not informative. Here we will resort to cuts of the cube ( e.g. cut $\left.\psi_{a}=\psi_{b}\right)$ and plot density and phase in such cuts.

The question is now where to cut. Clearly a knowledge of the classical flow would be most helpful here. In it's absence analysis as follows is also useful. The cuts should contain organizing elements about which the density is localized. The phase should be simple over the regions of the plane having high density. Note the idea of an organizing structure is that one has found the configuration part of the quasiregular resonant zone of the coupled modes. The phase simplicity can be used as a tool for finding organizing structures if they exist. Here we have used both trial and error as well as dynamics guidance to find the cuts.

Dynamics guidance means for example that in $\mathrm{CDBrClF}$ the resonance with closest frequency is $\omega_{a} \approx \omega_{b}$ and its associated $k_{a, b}$ in the Hamiltonian is largest. This suggests that modes a and b should be coupled in the plane $\psi_{a}=\psi_{b}+$ constant which assures $\omega_{a}^{e f f}=\omega_{b}^{e f f}$. Note the constant will vary as explained below in a dynamically meaningful way, i.e. it will be used for state classification on a ladder of states.

The Fermi choice $\omega_{s}=2 \omega_{a}$ along with $\omega_{s}=2 \omega_{b}$ would then give by similar reasoning the organizing line $\psi_{a}=c_{1}, \psi_{b}=c_{2}$. For some states several organizing structures could be used and the same dynamics revealed. Our experience is to choose those corresponding resonances which seem more important in $H$ and which give longer ladders of states.

\subsection{Scheme for a large number of states throughout the polyad}

Starting at the bottom of the polyad we find about 64 states that definitely lie in the Darling Dennison $\omega_{a}=\omega_{b}$ resonance class in that they have densities localized about a plane $\psi_{a}=\psi_{b}+$ constant albeit often with different constants. They also have simple phase plots in these planes. At the lower end a few of them could also be organized by the $\omega_{s}=2 \omega_{a}, \omega_{s}=2 \omega_{b}$ Fermi resonances, i.e. about a line in $f$ direction; but they occur when stretch excitation is zero and this classification is not very physical.

Some of the lowest states could also be assigned for all modes by quantum numbers $n_{j}$ and correspond to continuous deformations of basis functions. Although these $n_{j}$ are useful in the lift for obtaining actions these assignment misses the phase and frequency locking and the localization of the density. In addition we looked very carefully for primary tori in the classical phase space at the corresponding energy and did not find any. Therefore EBK quantization cannot be applied. And classical dynamics shows that the assignment by $n_{j}$ for $j=a, b, f$ is unphysical.

To illustrate our ideas let us consider two states, state $s=4$ and $s=46$ at the bottom and the middle of the ladder respectively. The former state is localized about the plane $\psi_{a}=\psi_{b}$ and the latter about $\psi_{a}=\psi_{b}+\pi / 2$ as seen in figures 1a and 1c for 
the former and figures $2 \mathrm{a}$ and $2 \mathrm{c}$ for the latter. Both localizations give the DD $\omega_{a}=\omega_{b}$ expectation. Note how only in the former case also the line $\psi_{a}=\pi / 2, \psi_{b}=\pi / 2$ could have been used as organizing center. In the diagrams all features come in double because of the symmetry mentioned at the end of section 4 . Figures 1 and 2 show that if one stays on the organizing plane and heads say in the f direction the torus is looped during a $2 \pi$ change of $\psi_{f}$. This says that $\psi_{f}$ motion is free and that on the phase plot following any path that along which $\psi_{f} \rightarrow \psi_{f}+2 \pi$ the free $\exp \left(i n_{f} \psi_{f}\right)$ factor in the wave function should go to $\exp \left(i n_{f}\left(\psi_{f}+2 \pi\right)\right)$. In Fig.1b or Fig.2b respectively as $\psi_{f} \rightarrow \psi_{f}+2 \pi$ and if we count the revolutions along a line in $\mathrm{f}$ direction one sees a phase advance of $2 \pi$ and $10 \pi$ respectively. We conclude that $n_{f}=1$ for state 4 ( Fig.1b) and $n_{f}=5$ for state 46 ( Fig.2b). Sometimes here $n_{f}$ will be usefully called $l_{f}$ to emphasize that it is a longitudinal quantum number obtained by following the path of highest density around the f loop.

The phase change accompanying a loop of the a direction in figures $1 \mathrm{~b}$ and $2 \mathrm{~b}$ by the nature of the cut causes a looping on the $\psi_{a}=\psi_{b}+$ constant plane in the complete configuration space. This looping advances both $\psi_{a}$ and $\psi_{b}$ by $2 \pi$ and on this plane the wave function must change as $\sum c \exp (i \vec{n} \cdot \vec{\psi}) \rightarrow \sum c \exp \left(i\left(\vec{n} \vec{\psi}+2 \pi\left(n_{a}+n_{b}\right)\right)\right.$. The choice of the $\psi_{a}=\psi_{b}+$ constant plane required that we see a simple plane wave factor $\exp \left(i\left(n_{a} \psi_{a}+n_{b} \psi_{b}\right)\right)$ which means that all the important basis states with large mixing coefficients must have this type factor. Hence the wave function phase must advance as we sweep $\psi_{a}$ in this organizing plane by $2 \pi\left(n_{a}+n_{b}\right)$. We denote this by $l_{a+b}, l$ for longitudinal, i.e. motion along the organizing center as opposed to transverse to it. This longitudinal quantum number clearly tells us the total number of quanta in the Darling Dennison lock.

For state 4 in figure $1 \mathrm{~b} l_{a+b}=9$ and for state 46 in figure $2 \mathrm{~b} l_{a+b}=5$. Using the polyad expression for the stretch mode we get $n_{s}=P-\left(n_{f}-l_{a+b}\right) / 2=0$. Now a partial ( three out of four needed quantum numbers ) assignment can be made as $\left(P, l_{f}=n_{f}, n_{s}\right)$ or equivalently $\left(l_{a+b}, l_{f}=n_{f}, n_{s}\right)$. State 4 is now $(5,1,0)$ or $(9,1,0)$ respectively and state 46 is $(5,5,0)$ in both schemes as recorded in table I. In principle $n_{a}$ and $n_{b}$ no longer exist as good quantum numbers except when as explained these numbers represent conserved integer loop integrals as in the continuous deformation case for state 4 where both phase diagrams ( Figs 1b and 1d) look as they represent $2 \mathrm{D}$ plane waves. Figure $1 \mathrm{~d}$ then tells us that $n_{a}=0$ and $n_{b}=9$. This is useful only for obtaining $I_{a}=n_{a}+1 / 2=1 / 2$ and $I_{b}=n_{b}+1 / 2=9 / 2$ which will in turn be useful in the lift process.

For state 46 phase simplicity only happens in the organizing plane. Fig. 2d shows no useful phase information. The plot shows jumps along lines and ramp singularities where the phase value depends on the direction of approach.

At this point and in a similar way a full 64 states for $P=5$ can be assigned by $n_{s}$ and $n_{f}$. Actually another 32 seem to resemble this picture. The resemblance is only seen as trends from the systematic following of the 64 other ones. For these increasing but still smaller effects of many other resonances are taking their toll and they significantly degrade the assignable states into unassignable more heavily mixed quantum analogues of classical chaos. The table I indicates which states have less certain assignments. 
Certain of the states will be marked in the table as demixed states and given in $s / s^{\prime}$ notation, i.e. 50/51. For such pairs it will be seen that the eigenstates involved are nearly degenerate and usually do not have the features of any of the states on any of the ladders we identify. The near degeneracy is an indication on that possibly two ( noneigen ) states exist with even more degenerate energies that do fit on ladders but fail to appear due to mixing by accidental degeneracy. By assuming for such eigenstates various weighted mixings the demixed dynamically characterizable states can be recognized and included in the ladders.

For further simplifying and dynamical classification the states of the DD ladder can now be sorted by common values of $n_{f}$ and $n_{s}$ ( or equivalently $l_{a+b}$ and $l_{f}$ ), see table I. Clearly one quantum number is missing. In a lower dimensional problem ( e.g. [1]) in a similar case of DD lock ( there between local stretch modes $\mathrm{n}$ and $\mathrm{m}$ ) the longitudinal $l_{n+m}$ which was determined by counting phase advances along an organizing line in the $\psi_{m}=\psi_{n}$ direction, was complemented by counting nodes in the direction transverse to the organizing line by using this number for a transverse quantum number $t$. Here only remnants if any of these nodes are seen. The greater mixing in higher dimension wipes out this clarifying element. What does remain common is that states with the same other quantum numbers as above can be ordered by how far significant density extends into the direction transverse to the organizing plane. For the DCO this gives the same $t$ ordering.

Here we adopt this criterion to organize the states in the $l_{f}, l_{a+b}$ or $n_{f} n_{s}$ subladders and also observe in Table I that this ordering simultaneously gives an energy ordering. This $t$ ordering number is our final quantum number. Additional support for our classification comes from the classical mechanics where trajectories at eigenstate energies flowing along the organizing plane fluctuate further in the transverse direction the greater is $t$. Most gratifyingly each subladder has an energy spectrum that is quite reminiscent of an anharmonic oscillator. As such locally and physically for each subladder of fixed $l_{f}$ and $l_{a+b}$ but varying $t$ we envision a dynamic potential causing the system to remain near the organizing structure. The levels of this potential are the states of the subladders.

Even at this stage where other ladders discussed below are omitted spectral complexity arises from the interlacing of energy levels of different subladders. The roughly 96 states on the ladder which only demands the DD-a/b lock for its construction gives a complex spectrum and nontransparent energy spacings.

The reader will note that some ladder states in table I are missing. These states could not be found but their disappearance is confirmed using the anharmonic models expected energy spacing. Formally these states lie among the highly mixed ( chaotic ? ) states we discuss below. They are mixings of more than two dynamically identifiable states and do not show the near degeneracy that our demixing process identifies.

The actions $I_{j}$ can be gotten for the modes s and f using $I_{j}=n_{j}+1 / 2$, in addition we have $I_{a}+I_{b}=l_{a+b}+1$. Then $I_{a}=J_{a}$ and $I_{b}=J_{b}$ is gotten from the state functions using Eq.31. To observe approximate averaged motion of the Deuterium atom the $I_{j}$ can be used in Eq.32 for $\theta$ to enable us to get $I(t)$ and $\phi(t)$ and then using Eq. 33 to draw qualitatively pictures for the various pairs $\left(q_{j}, p_{j}\right)$. Actually a great deal can be 
said without the integration for $\theta$. In $\left(q_{s}, q_{a / b / f}\right)$ planes a roughly rectangular region is seen whose sides are proportional to $\sqrt{2 I_{s}}$ and $\sqrt{2 I_{a / b / f}}$. The deuterium trajectories motion interior to the rectangle will be quasiperiodic motion. How the exact trajectory depends on the initial choices of $\phi_{s}$ and $\psi_{a / b / f}$ and is really not important. In the $\left(q_{a}, q_{b}\right)$ plane organization structure leads to ellipses whose relative ranges ( excursion from zero displacement $)$ in the $\left(q_{a}, q_{b}\right)$ variables is $\sqrt{I_{a} / I_{b}}$. The ellipses eccentricity depends on the organizing structures phase shift, viz. $\psi_{a}=\psi_{b}+\delta \rightarrow \phi_{a}=\phi_{b}+\delta$. $\delta=0$ and $\pi$ give zero angular momentum and something approaching a straight line motion while $\delta= \pm \pi / 2$ gives maximal angular momentum and elliptical motion which becomes circular if $I_{a}=I_{b}$. The $t$ value is the out of phase motion and causes the trajectory to change slowly its eccentricity.

\subsection{Scheme for the upper end of the polyad}

At the upper end of the polyad we take to start states 160 and 161. A cut in $\psi_{b}=0$ ( or any other value of $\psi_{b}$ ) reveals as we see in Fig.3c for state 160 what looks like two columns of density localized around $\psi_{a}=\pi$ and $\psi_{f}=\pi$. In comparison state 161 ( not shown ) only has one column. A cut in the plane $\psi_{b}=$ constant is required to see that the columns really exist. Figure 3c shows that they do for state 160 and it also holds for state 161. Clearly for both these states the line $\psi_{a}=\pi, \psi_{f}=\pi$ is the organizing structure. Since it loops in $\psi_{b}$ mode $\mathrm{b}$, is decoupled and the phase picture for both states shows ( figure $3 \mathrm{~b}$ for state 160 , state 161 not shown ) that as $\psi_{b} \rightarrow \psi_{b}+2 \pi$ along the organizing line no wave fronts are crossed and hence there is no phase advance. As such $l_{b}=n_{b}=0$. For the upper end of the polyad also classical dynamics shows a flow in $\mathrm{b}$ direction.

Now the $\psi_{a}=\psi_{f}=\pi$ organizing structure implies that the phase locks are $\phi_{a}=$ $\phi_{s} / 2+\pi$ and $\phi_{f}=\phi_{s} / 2+\pi$. Time differentiation of these relations show that the frequency locks are of 2:1 Fermi type with the stretch mode locking with the bend mode a and the mode $\mathrm{f}$. Clearly for the organizing structure $\psi_{a}=\psi_{f}$ or equivalently then $\phi_{a}=\phi_{f}$ is a valid phase lock and $\omega_{a}=\omega_{f}$ is a DD 1:1 frequency lock. Since all three types of terms appear in the Hamiltonian we can be assured that all three frequencies are in lock and that the quantum numbers $n_{a}, n_{f}$ and $n_{s}$ no longer exist. They must be replaced by three quantum numbers of the lock one of which can be $\mathrm{P}$ and the other two can be taken as the number of nodes seen in the $\psi_{b}=0$ cut along the antidiagonal as $t_{1}=1$ and along the diagonal as $t_{2}=0$. For state 161 since one column exists $t_{1}=t_{2}=0$.

Table II shows the assignment for the states whose analysis is based on the same organizing structure. Figure 3a shows a vertical cut into the cube along the antidiagonal of figure 3c. It reveals at $\psi_{a}=\pi$ a vertical nodal line corresponding to the node on the antidiagonal line $\psi_{f}=-\psi_{a}$ in figure 3c. The assignment of states 161 and 160 in table II is complete. $P-n_{b}$ is the number of excitations in the three way lock and the quantum numbers $t_{1}$ and $t_{2}$ tell us how much of this excitation is transverse to the columns and therefore is a measure of the extension of the density outward from the organizing line created by any two of the three resonances mentioned above, only two 
of them are independent.

All entries in table II use the same organizing structure as these states. Two of them needed to be demixed before being assigned. They are not continuous deformations of basis states ( the phase functions never look like plane waves ), only the action $I_{b}$ can be obtained as $I_{b}=n_{b}+1 / 2$. Estimates of $I_{a}, I_{f}$ and $I_{s}$ must be gotten from computing the quantum state average of $J_{a}, J_{f}$ and by the canonical transformations.

An idea of the internal motion of the deuterium and the CF stretch can be obtained from the actions, phase relations and the $t_{1}$ and $t_{2}$ values. In all $\left(q_{i}, q_{j}\right)$ planes the actions again define the maximal displacement $\sqrt{2 I_{j}}$ of each $q_{j}$ from its equilibrium. Clearly this range is meant in the sense that an average is made over the values of the two variables not represented in the plane. The planes $\left(q_{b}, q_{j}\right), j=a, f, s$ will show rectangularly bound quasiperiodic motion. In the $\left(q_{s}, q_{a / f}\right)$ plane a $\mathrm{U}$ shaped region is swept out. Here $q_{a / f}$ reaches its extreme values as $q_{s}$ reaches its maximum displacement and $q_{s}$ sweeps its range twice for each sweep of $q_{a / f}$. Increasing $t_{1}$ and/or $t_{2}$ causes the lifted trajectory to oscillate in a tube about this basic U lift. Of course $q_{f}$ and $q_{a}$ reach their maxima ( minima) in phase as the three modes $\mathrm{s}$, a and $\mathrm{f}$ are phase locked.

\subsection{Patterns in the dense region of the polyad}

In the middle of the polyad there are approximately 50 states with very complicated wave functions which we could not classify. It is possible that further and closer analysis could reveal some systematics. These states are dispersed among the states mentioned in subsection 7.1. But interestingly a few simple states which do not fall into any scheme recognized are dispersed in this region. In this subsection we present a few such states.

State 111 has its density concentrated in the plane $\psi_{f}=\psi_{b}$ in which plane the phase function also comes close to a plane wave with $l_{a}=0$ and $l_{b+f}=10$ ( see parts $\mathrm{a}$ and $\mathrm{b}$ of figure 4 ); this simple $n_{s}=0$. In the density plot we see a strong tendency towards a-b coupling but it is not perfect. The parts $\mathrm{c}$ and $\mathrm{d}$ of the figure show density and phase in the transverse plane $\psi_{f}=\pi / 2$. We see a very good concentration of the density along the line $\psi_{b}=\psi_{f}$ indicating $t=0$ with respect to this organizing plane. Therefore in this state all excitation is in the in phase combined DD-b/f ( bend of deuterium and stretch of fluorine ) motion. The degrees of freedom a and s are not excited and the assignment is complete.

Numerical results for state 103 are shown in figure 5, part a and b show density and phase in the plane $\psi_{f}=0$ which is the plane of high density and thereby the organizing structure. Part $\mathrm{c}$ and $\mathrm{d}$ show density and phase in the transverse plane $\psi_{a}=\pi / 2$. We clearly see how the density is concentrated around the planes $\psi_{f}=0 \mathrm{or} \pi$. In the plane of high density the phase function comes close to a plane wave with $l_{a}=1$ and $l_{b}=4$. A few other states ( e.g. 108 and 148 ) fall into the same scheme. For the lifted motion in the displacement space we can draw the following conclusions: a and b both run freely with an excitation corresponding to their loop numbers read off from the phase function in the plane $\psi_{f}=0, \mathrm{f}$ is completely coupled to $\mathrm{s}$ in a 2:1 Fermi resonance. $\mathrm{P}=5$ and the $t=0$ from figure $5 \mathrm{c}$ complete the assignment. 
State 140, Figure 6, is an example with a concentration of the density around a point, in this particular case the point $\psi_{a}=-\pi / 2, \psi_{b}=\pi / 2, \psi_{f}=\pi / 2$. This means coupling of all degrees of freedom at a particular phase value. The phase function is rather simple globally and indicates $n_{a}=0, n_{b}=0, n_{f}=8$. Because of polyad conservation we then conclude that $n_{s}=1$. This means that all four actions are $I_{j}=n_{j}+1 / 2$. Accordingly we have a coupled 2:1 Fermi-s/f motion whereas the formal coupling of $\mathrm{a}$ and $\mathrm{b}$ with $\mathrm{s}$ is rather irrelevant since their excitation numbers are 0 . This interpretation is based on the phase function even though the density is concentrated around a point, where logically we should classify states by transverse excitations in 3 directions ( are all zero in this particular state).

\section{Conclusions}

We have shown how our previously developed methods can also be applied to systems with 3 degrees of freedom after reduction. For seventy percent of the states the strategy works successfully. We investigate the wave functions constructed on a toroidal configuration space. We investigate on which subsets the density is concentrated and evaluate the phase function on this subset of high density. This allows us to obtain a set of excitation numbers for the states; in the best cases we obtain a complete set of excitation numbers which provide a complete assignment of the state. The excitation numbers can be interpreted as quasi conserved quantities for this particular state.

The success of the method is based on 3 properties of our strategy which more traditional approaches starting from potential surfaces do not have. First, we start from an algebraic Hamiltonian which naturally decomposes into an unperturbed $H_{0}$ and a perturbation $W$. Perturbation theory assures us that $W$ contains only the dominant interactions, leaving out those that do not affect the qualitative organization of the states. In addition having the ideal $H_{0}$ results in the classical Hamiltonian being formulated from the start in action and angle variables belonging to $H_{0}$. For a potential surface it is very complicated to construct an appropriate $H_{0}$ and the corresponding action/angle variables. Our knowledge of the correct $H_{0}$ allows the continuous deformation connection of some eigenstates of $H$ with the corresponding eigenstates of $H_{0}$ which was useful for many states. Second in the algebraic Hamiltonian the polyad ( which for the real molecule is only an approximate conserved quantity ) is an exactly conserved quantity and allows the reduction of the number of degrees of freedom. We always deal with one degree of freedom less than the number of degrees of freedom on the potential surface. Third, our strategy depends to a large extent on having a compact configuration space with nontrivial homology where the phase of the wave function is important and where the phase advances divided by $2 \pi$ along the fundamental cycles of the configuration space provide a large part of the excitation numbers. In part also feature 1 is responsible for the importance of the phases. This is an essential difference to working in usual position space. In total we have found a semiclassical representation of the states which is actually much simpler to view than the usual position space one. 
The classical dynamics of the system shows rather little regular motion and is strongly unstable. Then the question arises in which way the quantum states reflect anything from the classical chaos. We propose the following answer: In the middle of the polyad a large number of states do not show any simple organization pattern. For some of those states it might be that we simply have not found the right representation in which to see the organizational center. However we doubt that this is the case for many states. For many of the complicated states the wave function is really without a simple structure, it seems to be a complicated interference between several organizational structures. Then immediately comes the idea to mix these states with their neighboring states to extract simple structures in the way as we did it for states 50 and 51. For most of the complicated states this method does not lead to any success. This indicates that underlying simple patterns are distributed over many states so that demixing becomes rather hopeless and maybe also meaningless. In the spirit of reference [19] this indicates that the density of states is already so high that many states fall into the energy interval over which states are considered almost degenerate and mix strongly. This is a strong difference to the systems we have investigated before which could be reduced to 2 degrees of freedom. There the density of states was much lower such that mixing only occurred occasionally between 2 neighboring states. This stronger mixing gives some clue as to how the quantum wave functions become complicated for classically chaotic systems and for sufficiently high quantum excitation.

Finally there may come up the question why we do not use some of the common statistical procedures ( like e.g. random matrix theory ) to treat our system when effects of classical chaos become evident in a quantum system. The answer is: We are not satisfied with only generic (universal) properties of the system or with the investigation to which extent such universal properties are realized by our system. We aim to investigate each individual quantum state one after the other and to work out its individual properties in order to arrive in the best case at a complete set of quantum numbers in addition at a detailed description of the atomic motion underlying the individual state. Previous examples have shown that our procedure provides a complete classification for simpler systems which can be reduced to 2 degrees of freedom. As the example presented in this paper shows, this program is doable with good success for a large number of states even in a system with originally 4 degrees of freedom, reducible only to 3 degrees of freedom and with a complicated classical dynamics.

\section{Acknowledgments}

We thank Dr. E. Atilgan for helpful discussions. CJ thanks CONACyT for support under grant number 33773-E and DGAPA for support under grant number IN-109201. HST thanks the US Department of Energy, Chemical Science Division for support under grant number DE-FG03-01IR15147. 


\section{Table I}

Table I: This table gives the classification and assignment of all states organized around planes $\psi_{b}=\psi_{a}+$ constant. The first column gives the number of the state ordered according to energy. If the structure only becomes clear after demixing, then it contains in addition the number(s) of the state(s) with which it is demixed. The second column gives the energy in units of $\mathrm{cm}^{-1}$. The third and fourth columns give the two longitudinal quantum numbers $l_{f}$ and $l_{a+b}$. The fifth column gives the transverse quantum number $t$. For such states where the individual quantum numbers $n_{a}$ and $n_{b}$ exist, these are given in columns 6 and 7 . If these phase functions show some first indications of developing singularities, these quantum numbers are put in brackets. States where the pattern is not so clear but where the general trends suggest the assignment have a $*$ in the last column. The total assignment is $\left(\mathrm{P}, l_{f}\right.$ or $n_{f}, l_{a+b}$ or $\left.n_{s}, \mathrm{t}\right)$.

\begin{tabular}{c|c|c|c|c|c|c|c} 
Number(s) & Energy & $l_{f}=n_{f}$ & $l_{a+b} / n_{s}$ & $t$ & $n_{a}$ & $n_{b}$ & \\
\hline 1 & 8908 & 0 & $10 / 0$ & 0 & 0 & 10 & \\
2 & 8997 & 0 & $10 / 0$ & 1 & 1 & 9 & \\
3 & 9080 & 0 & $10 / 0$ & 2 & 2 & 8 & \\
5 & 9156 & 0 & $10 / 0$ & 3 & 3 & 7 & \\
7 & 9226 & 0 & $10 / 0$ & 4 & 4 & 6 & \\
10 & 9290 & 0 & $10 / 0$ & 5 & $(5)$ & $(5)$ & \\
13 & 9348 & 0 & $10 / 0$ & 6 & $(6)$ & $(4)$ & \\
16 & 9400 & 0 & $10 / 0$ & 7 & & & \\
20 & 9446 & 0 & $10 / 0$ & 8 & & & \\
$25 / 24$ & 9485 & 0 & $10 / 0$ & 9 & & & $*$ \\
$29 / 28 / 30$ & 9526 & 0 & $10 / 0$ & 10 & & & $*$
\end{tabular}




\begin{tabular}{|c|c|c|c|c|c|c|c|}
\hline Number(s) & Energy & $l_{f}=n_{f}$ & $l_{a+b} / n_{s}$ & $t$ & $n_{a}$ & $n_{b}$ & \\
\hline 4 & 9107 & 1 & $9 / 0$ & 0 & 0 & 9 & \\
\hline 6 & 9184 & 1 & $9 / 0$ & 1 & 1 & 8 & \\
\hline 8 & 9254 & 1 & $9 / 0$ & 2 & 2 & 7 & \\
\hline 11 & 9317 & 1 & $9 / 0$ & 3 & (3) & $(6)$ & \\
\hline 14 & 9373 & 1 & $9 / 0$ & 4 & (4) & $(5)$ & \\
\hline 18 & 9423 & 1 & $9 / 0$ & 5 & (5) & (4) & \\
\hline 22 & 9466 & 1 & $9 / 0$ & 6 & (6) & $(3)$ & \\
\hline $27 / 26$ & 9507 & 1 & $9 / 0$ & 7 & (7) & $(2)$ & \\
\hline $33 / 34$ & 9555 & 1 & $9 / 0$ & 9 & & & \\
\hline 9 & 9278 & 2 & $8 / 0$ & 0 & 0 & 8 & \\
\hline 12 & 9343 & 2 & $8 / 0$ & 1 & (1) & $(7)$ & \\
\hline 17 & 9402 & 2 & $8 / 0$ & 2 & & & \\
\hline 21 & 9452 & 2 & $8 / 0$ & 3 & (3) & $(5)$ & \\
\hline $26 / 27$ & 9493 & 2 & $8 / 0$ & 4 & (4) & (4) & \\
\hline $31 / 32$ & 9544 & 2 & $8 / 0$ & 5 & & & $*$ \\
\hline 35 & 9579 & 2 & $8 / 0$ & 6 & & & \\
\hline $41 / 42$ & 9623 & 2 & $8 / 0$ & 7 & & & \\
\hline 19 & 9428 & 3 & $7 / 0$ & 0 & (0) & $(7)$ & \\
\hline $24 / 25$ & 9479 & 3 & $7 / 0$ & 1 & (1) & $(6)$ & \\
\hline $28 / 29 / 30$ & 9513 & 3 & $7 / 0$ & 2 & & & $*$ \\
\hline 36 & 9585 & 3 & $7 / 0$ & 3 & & & \\
\hline $42 / 41$ & 9634 & 3 & $7 / 0$ & 4 & (4) & $(3)$ & \\
\hline 44 & 9659 & 3 & $7 / 0$ & 5 & & & \\
\hline $48 / 49$ & 9681 & 3 & $7 / 0$ & 6 & & & \\
\hline $34 / 33$ & 9562 & 4 & $6 / 0$ & 0 & & & \\
\hline 43 & 9655 & 4 & $6 / 0$ & 2 & & & \\
\hline $51 / 50$ & 9717 & 4 & $6 / 0$ & 3 & & & \\
\hline 54 & 9740 & 4 & $6 / 0$ & 4 & & & \\
\hline $81 / 80$ & 9922 & 4 & $6 / 0$ & 6 & & & \\
\hline 46 & 9665 & 5 & $5 / 0$ & 0 & & & \\
\hline 61 & 9794 & 5 & $5 / 0$ & 2 & & & \\
\hline $65 / 67$ & 9822 & 5 & $5 / 0$ & 3 & & & \\
\hline 73 & 9879 & 5 & $5 / 0$ & 4 & & & \\
\hline $80 / 81$ & 9917 & 5 & $5 / 0$ & 5 & & & $*$ \\
\hline 60 & 9784 & 6 & $4 / 0$ & 0 & & & \\
\hline 72 & 9862 & 6 & $4 / 0$ & 1 & & & $*$ \\
\hline 77 & 9898 & 6 & $4 / 0$ & 2 & $(2)$ & $(2)$ & $*$ \\
\hline $81 / 80$ & 9922 & 6 & $4 / 0$ & 3 & & & $*$ \\
\hline 86 & 9967 & 6 & $4 / 0$ & 4 & & & $*$ \\
\hline 91 & 10008 & 6 & $4 / 0$ & 5 & & & $*$ \\
\hline 89 & 9993 & 7 & $3 / 0$ & 2 & $(2)$ & (1) & \\
\hline $95 / 96$ & 10047 & 7 & $3 / 0$ & 3 & & & \\
\hline
\end{tabular}




\begin{tabular}{c|c|c|c|c|c|c|c} 
Number(s) & Energy & $l_{f}=n_{f}$ & $l_{a+b} / n_{s}$ & $t$ & $n_{a}$ & $n_{b}$ & \\
\hline 97 & 10053 & 8 & $2 / 0$ & 1 & $(1)$ & $(1)$ & $*$ \\
101 & 10087 & 8 & $2 / 0$ & 2 & & & \\
$105 / 106$ & 10122 & 9 & $1 / 0$ & 1 & $(1)$ & $(0)$ & \\
15 & 9394 & 0 & $8 / 1$ & 0 & & & \\
23 & 9473 & 0 & $8 / 1$ & 1 & & & $*$ \\
$32 / 31$ & 9547 & 0 & $8 / 1$ & 2 & & & $*$ \\
40 & 9611 & 0 & $8 / 1$ & 3 & & & $*$ \\
47 & 9670 & 0 & $8 / 1$ & 4 & $(4)$ & $(4)$ & \\
52 & 9723 & 0 & $8 / 1$ & 5 & $(5)$ & $(3)$ & \\
59 & 9778 & 0 & $8 / 1$ & 6 & $(6)$ & $(2)$ & \\
64 & 9817 & 0 & $8 / 1$ & 7 & & & \\
38 & 9597 & 1 & $7 / 1$ & 0 & & & \\
45 & 9660 & 1 & $7 / 1$ & 1 & $(1)$ & $(6)$ & \\
$50 / 51$ & 9714 & 1 & $7 / 1$ & 2 & & & \\
58 & 9766 & 1 & $7 / 1$ & 3 & $(3)$ & $(4)$ & \\
63 & 9808 & 1 & $7 / 1$ & 4 & $(4)$ & $(3)$ & $*$ \\
$69 / 70$ & 9849 & 1 & $7 / 1$ & 5 & & & \\
74 & 9883 & 1 & $7 / 1$ & 6 & & & $*$ \\
56 & 9756 & 2 & $6 / 1$ & 0 & & & \\
62 & 9802 & 2 & $6 / 1$ & 1 & $(1)$ & $(5)$ & $*$ \\
71 & 9859 & 2 & $6 / 1$ & 2 & & & $*$ \\
$78 / 79$ & 9913 & 2 & $6 / 1$ & 3 & & & $*$ \\
83 & 9944 & 2 & $6 / 1$ & 4 & & & $*$ \\
87 & 9972 & 2 & $6 / 1$ & 5 & $(5)$ & $(1)$ & $*$ \\
$75 / 76$ & 9887 & 3 & $5 / 1$ & 0 & & & \\
90 & 10002 & 3 & $5 / 1$ & 2 & $(2)$ & $(3)$ & $*$ \\
94 & 10042 & 3 & $5 / 1$ & 3 & $(3)$ & $(2)$ & $*$ \\
$106 / 105$ & 10125 & 4 & $4 / 1$ & 2 & $(2)$ & $(2)$ & $*$ \\
120 & 10256 & 5 & $3 / 1$ & 2 & $(2)$ & $(1)$ & $*$ \\
127 & 10328 & 5 & $3 / 1$ & $?$ & & & $*$ \\
$132 / 133$ & 10384 & 6 & $2 / 1$ & $?$ & & & \\
$135 / 136$ & 10432 & 7 & $1 / 1$ & $?$ & & & $*$ \\
$70 / 69$ & 9854 & 0 & $6 / 2$ & 0 & & & \\
$92 / 93$ & 10013 & 0 & $6 / 2$ & 3 & & & \\
104 & 10115 & 0 & $6 / 2$ & 5 & $(5)$ & $(1)$ & \\
103 & 10094 & 1 & $5 / 2$ & 1 & $(1)$ & $(4)$ & $*$ \\
108 & 10136 & 1 & $5 / 2$ & 2 & $(2)$ & $(3)$ & $*$ \\
113 & 10177 & 1 & $5 / 2$ & 3 & $(3)$ & $(1)$ & $*$ \\
150 & 10215 & 1 & $5 / 2$ & 4 & & & $*$ \\
10233 & 1 & $5 / 2$ & 5 & & & $*$ \\
10588 & 4 & $2 / 2$ & $?$ & & & \\
10633 & 5 & $1 / 2$ & $?$ & & & $*$ \\
& & & & & &
\end{tabular}




\section{Table II}

Table II: This table gives the classification and assignment of all states organized around fibers in b direction. The first column gives the number of the state ordered according to energy. If the structure only becomes clear after demixing, then it contains in addition the number(s) of the state(s) with which it is demixed. The second column gives the energy in units of $\mathrm{cm}^{-1}$. The third column gives the longitudinal quantum number $l_{b}$. The fourth and fifth column give the transverse quantum numbers $t_{1}$ and $t_{2}$.

\begin{tabular}{c|c|c|c|c} 
Number(s) & Energy & $l_{b}$ & $t_{1}$ & $t_{2}$ \\
\hline 161 & 11096 & 0 & 0 & 0 \\
160 & 10953 & 0 & 1 & 0 \\
159 & 10917 & 1 & 0 & 0 \\
$158 / 157$ & 10834 & 1 & 1 & 0 \\
$157 / 158$ & 10818 & 0 & 2 & 0 \\
156 & 10768 & 1 & 2 & 0 \\
155 & 10730 & 0 & 0 & 1 \\
154 & 10720 & 1 & 3 & 0 \\
153 & 10706 & 0 & 1 & 1
\end{tabular}




\section{References}

[1] C. Jung, H. S. Taylor, and E. Atilgan, J. Phys. Chem. A 1063092 (2002).

[2] H. Waalkens, C. Jung, and H. S. Taylor, J. Phys. Chem. A 106911 (2002).

[3] C. Jung, E. Ziemniak, and H. S. Taylor, J. Chem. Phys. 1152499 (2001).

[4] C. Jung, H. S. Taylor, and M. P. Jacobson, J. Phys. Chem. A 105681 (2001).

[5] M. P. Jacobson, C. Jung, H. S. Taylor, and R. W. Field, J. Chem. Phys. 111600 (1999).

[6] A. Treollsch, and F. Temps, Zeitschrift Physikalische Chemie 215207 (2001).

[7] J. L. Teffo, V. I. Perevalov, and O. M. Lyulin, J. Mol. Spec. 168390 (1994).

[8] A. Beil, D. Luckhaus, and M. Quack, Ber. Bunsenges. Phys. Chem. 1001853 (1996).

[9] A. Beil, D. Luckhaus, M. Quack, and J. Stohner, Ber. Bunsenges. Phys. Chem. 101311 (1997).

[10] A. Beil, D. Luckhaus, R. Marquardt, and M. Quack, J. Chem. Soc. Faraday Discuss. 9949 (1994)

[11] M. Abbouti Temsamani, M. Herman, S. A. B. Solina, J. P. O'Brien, R. W. Field, J. Chem Phys. 10511357 (1996).

[12] M. P. Jacobson, J. P. O’Brien, R. J. Sibley, and R. W. Field, J. Chem. Phys. 109 121 (1999).

[13] A. Beil, H. Hollenstein, O. Monti, M. Quack and J. Stohner, J. Chem. Phys. 113 2701 (2000).

[14] E. L. Siebert III, and A. B. McCoy, J. Chem. Phys. 105469 (1996).

[15] M. Joyeux, J. Chem. Phys. 1092111 (1998).

[16] L. E. Fried, and G. S. Ezra, J. Chem Phys. 866270 (1987).

[17] M. Joyeux, S. C. Farantos and R. Schinke, J. Phys. Chem. A 106, 5407 (2002)

[18] S. Saini, J. Zakrewoki and H.S. Taylor, Phys. Rev. A 38390 (1988).

[19] L. Benet, T. H. Seligman and H. A. Weidenmüller, Phys. Rev. Lett. 71, 529 (1993) 

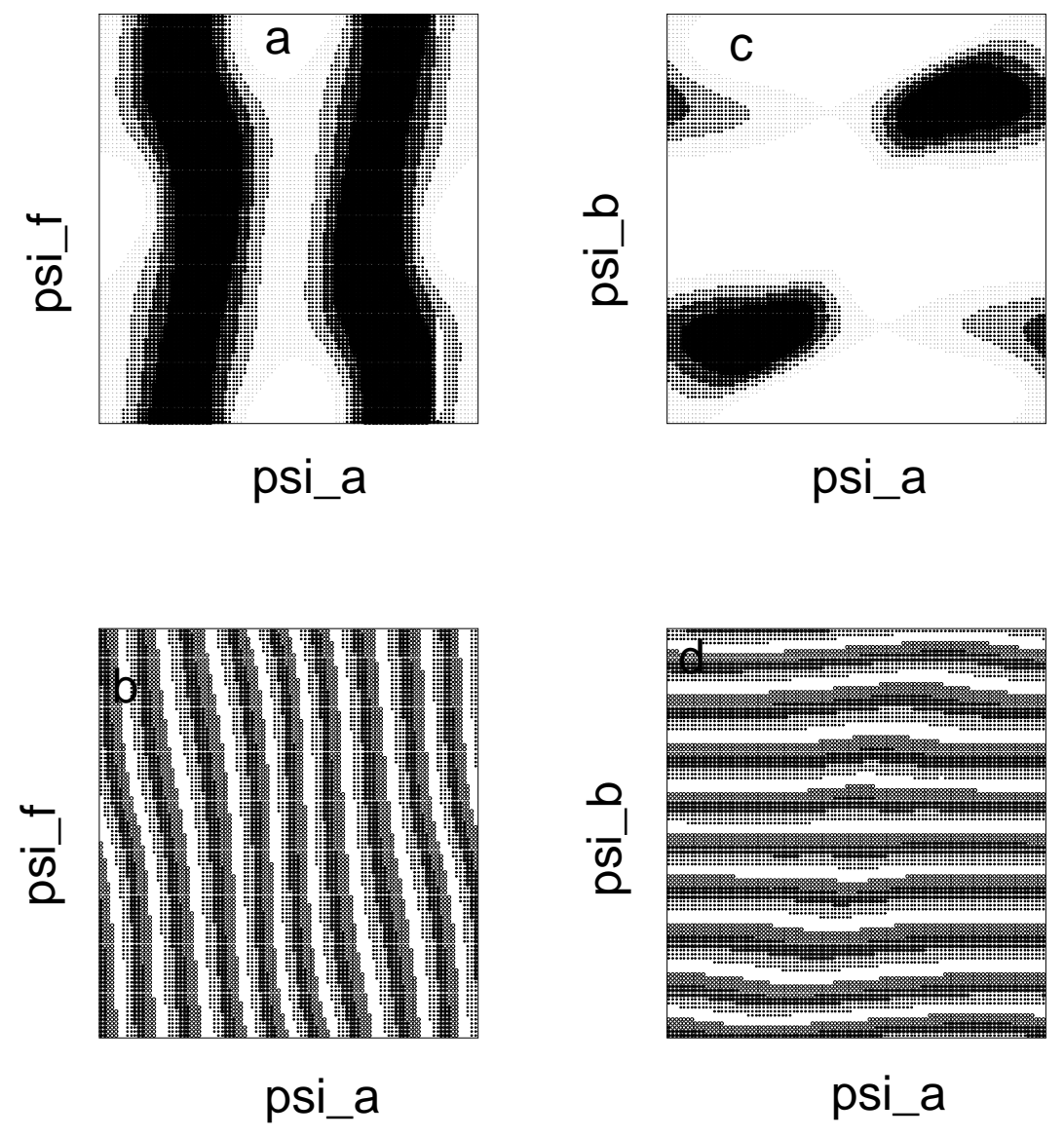

Figure 1: Plots of density ( part a ) and phase ( part b ) respectively in the plane $\psi_{b}=\psi_{a}$ and of density ( part c ) and phase ( part d ) respectively in the plane $\psi_{f}=0$ for the state 4 . In parts a and c darker grey means higher density. In parts $b$ and $d$ points with positive real part of the wave function are marked by a dot and points with a positive imaginary part of the wave function are marked by an open circle. All frame boundaries run from 0 to $2 \pi$ 

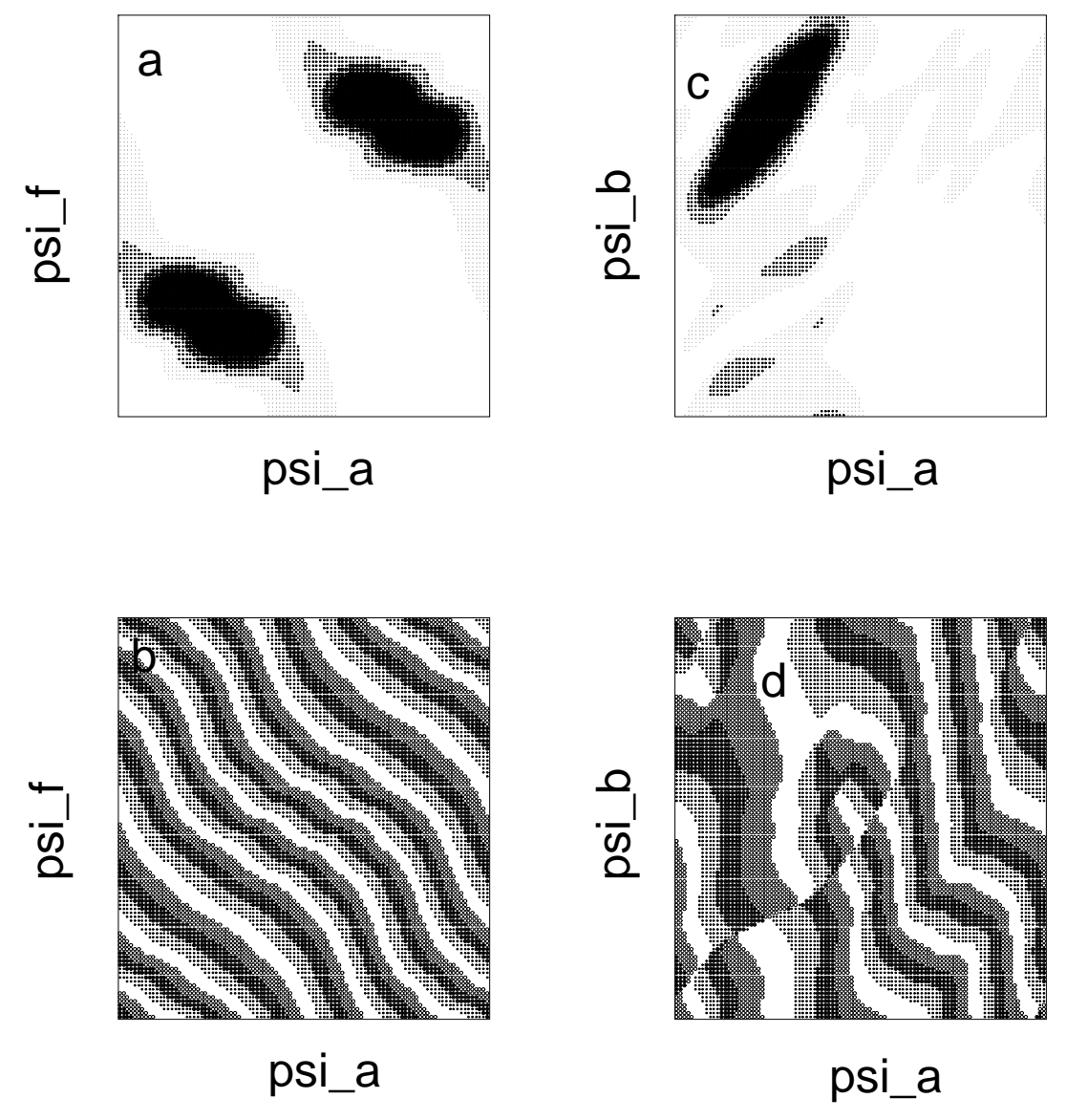

Figure 2: Plots for state 46 in the plane $\psi_{b}=\psi_{a}+\pi$ ( parts a and b ) and in plane $\psi_{f}=\pi / 2$ ( parts c and $\mathrm{d}$ ). Otherwise this plot is done in analogy to Fig.1. 

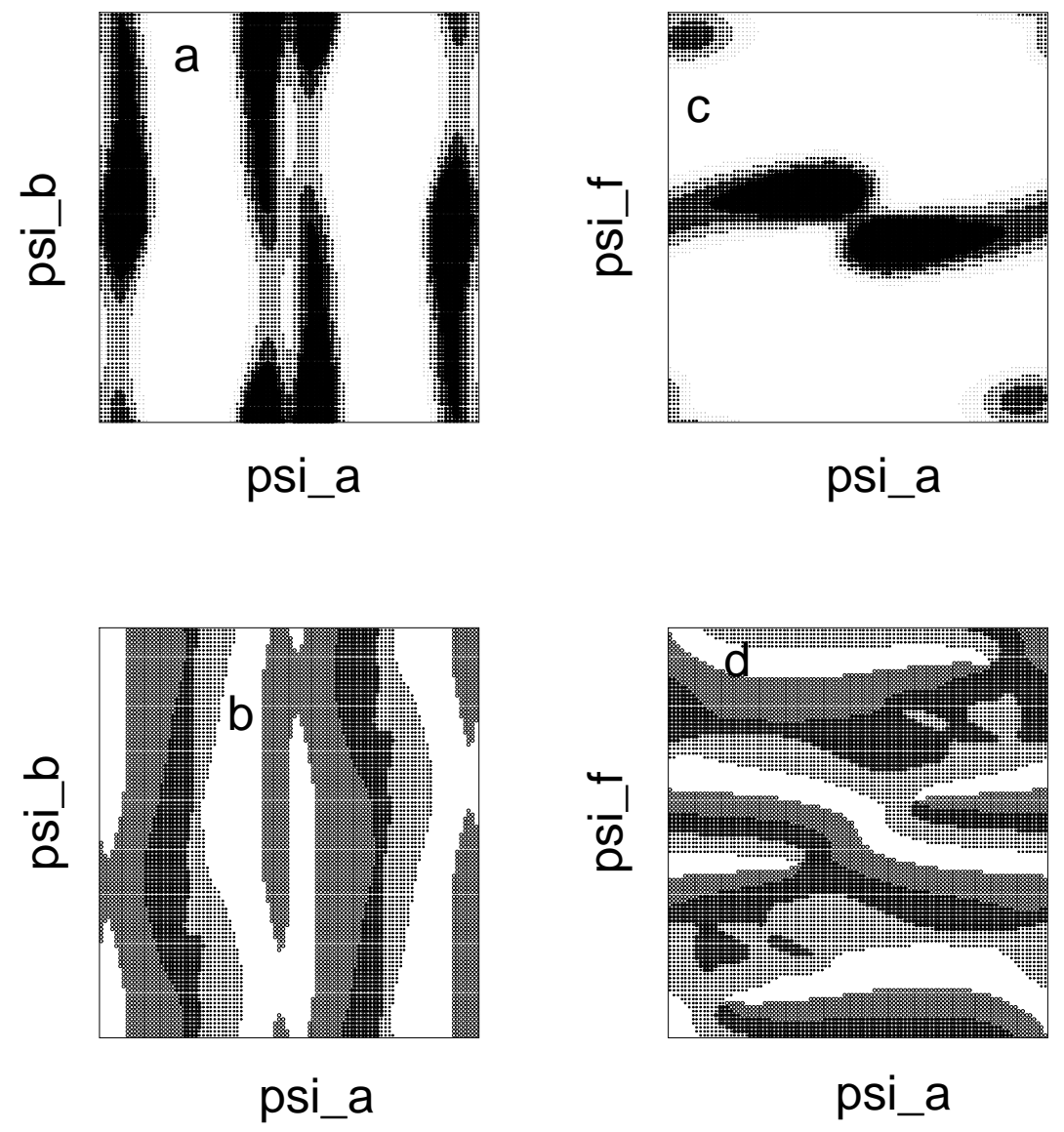

Figure 3: Plots for state 160 in the plane $\psi_{f}=-\psi_{a}$ ( parts a and b ) and in plane $\psi_{b}=0$ ( parts c and d ). Otherwise this plot is done in analogy to Fig.1. 

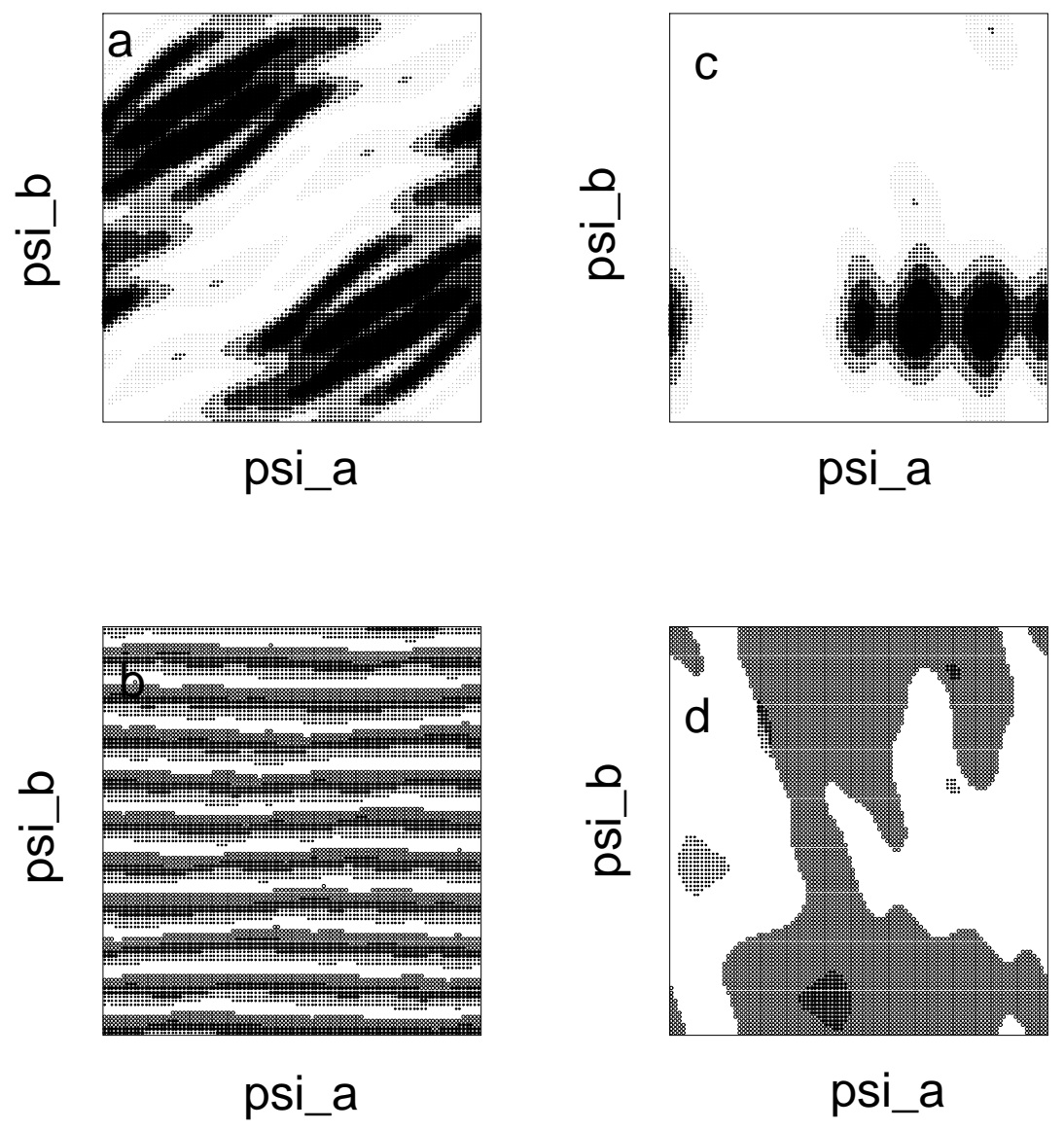

Figure 4: Plots for state 111 in the plane $\psi_{f}=\psi_{b}$ ( parts a and b ) and in plane $\psi_{f}=\pi / 2$ ( parts c and $\mathrm{d}$ ). Otherwise this plot is done in analogy to Fig.1. 

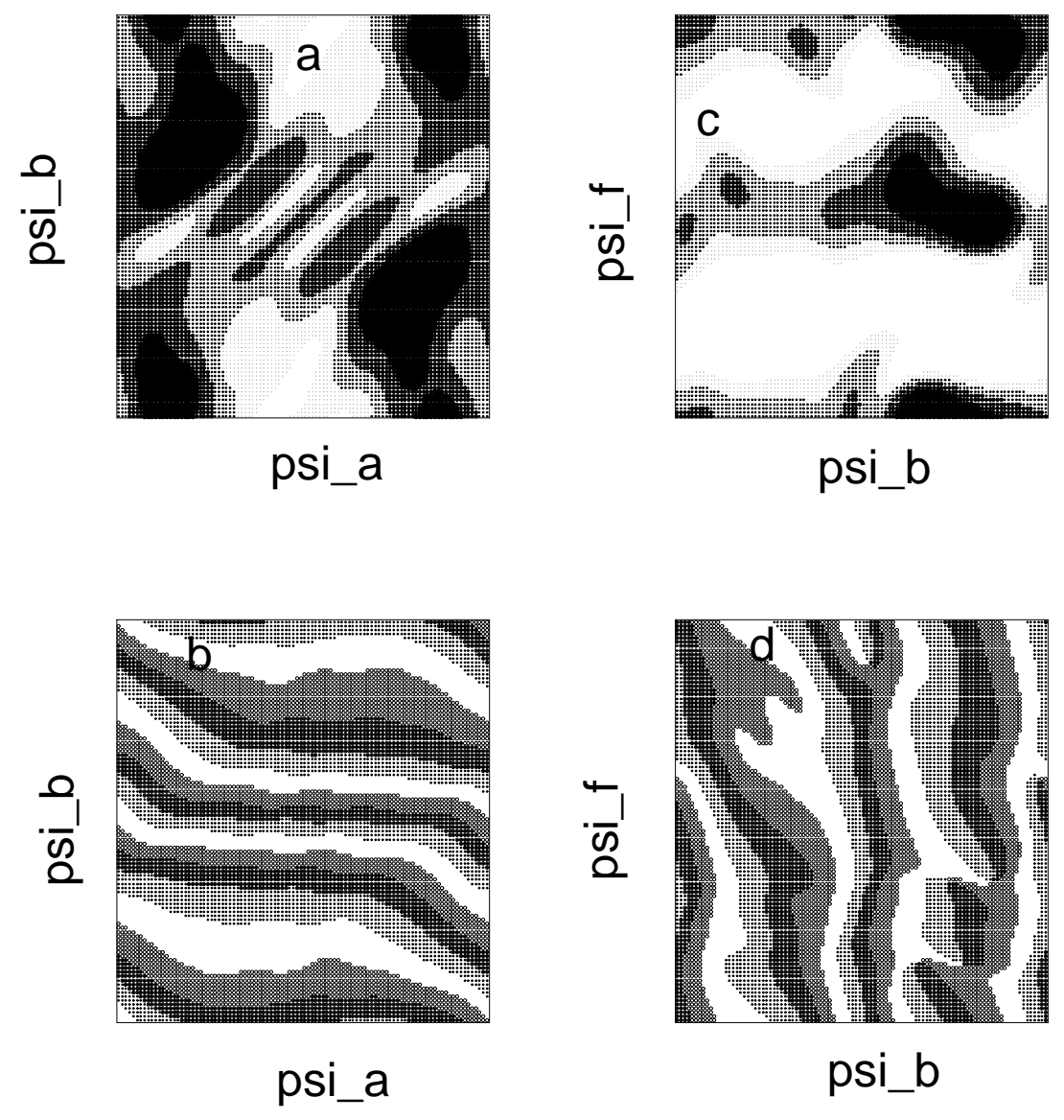

Figure 5: Plots for state 103 in the plane $\psi_{f}=0$ ( parts a and b ) and in plane $\psi_{a}=\pi / 2$ ( parts $\mathrm{c}$ and $\mathrm{d}$ ). Otherwise this plot is done in analogy to Fig.1. 


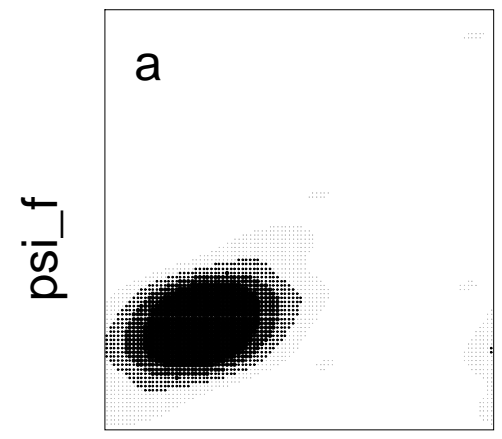

psi_b

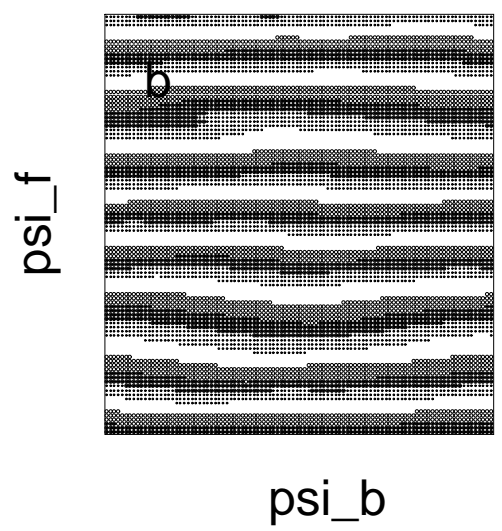

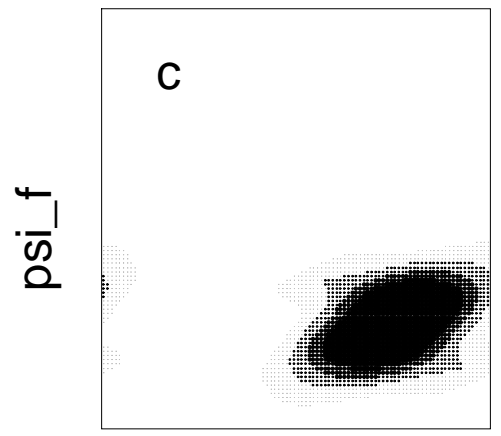

psi_a

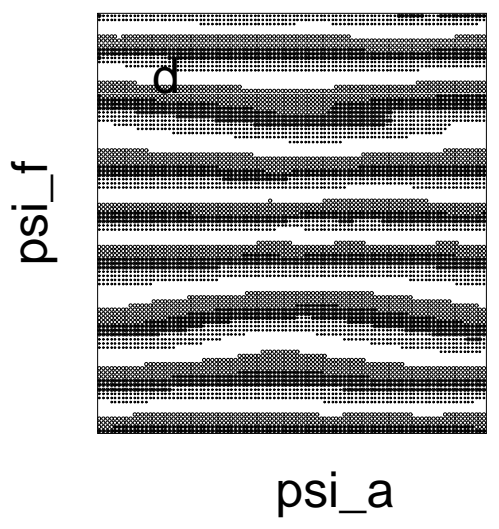

Figure 6: Plots for state 140 in the plane $\psi_{a}=3 \pi / 2$ ( parts a and b ) and in plane $\psi_{b}=\pi / 2$ ( parts c and d ). Otherwise this plot is done in analogy to Fig.1. 